\title{
A Rapid Method for Measuring Strontium-90 Activity in Crops in China
}

\author{
Lingjing Pan Pan ${ }^{1}$, Guobing Yu², Deyun Wen ${ }^{2}$, Zhi Chen ${ }^{1}$, Liusi Sheng ${ }^{1}$, Chung-King Liu ${ }^{1}$, and X. George Xu ${ }^{1,3 \star}$ \\ ${ }^{1}$ University of Science and Technology of China, Hefei, China \\ ${ }^{2}$ Environmental Radiation Surveillance Center, Environmental Protection Department of Anhui Province, Hefei, China \\ ${ }^{3}$ Rensselaer Polytechnic Institute, Troy, New York, USA
}

\begin{abstract}
A rapid method for measuring Sr-90 activity in crop ashes is presented. Liquid scintillation counting, combined with ion exchange columns 4", 4“(5“)-di-t-butylcyclohexane-18-crown-6, is used to determine the activity of Sr-90 in crops. The yields of chemical procedure are quantified using gravimetric analysis. The conventional method that uses ion-exchange resin with HDEHP could not completely remove all the bismuth when comparatively large lead and bismuth exist in the samples. This is overcome by the rapid method. The chemical yield of this method is about $60 \%$ and the MDA for Sr-90 is found to be $2.32 \mathrm{~Bq} / \mathrm{kg}$. The whole procedure together with using spectrum analysis to determine the activity only takes about one day, which is really a large improvement compared with the conventional method. A modified conventional method is also described here to verify the value of the rapid one. These two methods can meet different needs of daily monitoring and emergency situation.
\end{abstract}

\section{Introduction}

Sr-90 is an artificial radionuclide produced by spontaneous fission of $\mathrm{U}$ and $\mathrm{Pu}$ [1], which is associated with nuclear accidents, nuclear facility operations, and nuclear weapon explosion [2,3]. With historical nuclear explosion and accidents, a large number of radionuclides such as fission product strontium have been released to the atmosphere $[4,5]$. Due to its long half-life of about 28.5 years, it still exists in the environment. Once the fallouts were absorbed by crops, Sr-90 can eventually get into the human body and accumulate in the skeleton as the result of its chemical similarity with the calcium, and its daughter radionuclide Y-90 emits high-energy $\beta$ particles delivering a relatively high radiation dose to the bone and bone narrow tissues [6-7]. Thus it's extremely critical to determine the activity of Sr-90 in crops. As China is rapidly developing the nuclear power industry, establishing associated daily monitoring and emergency determining systems of Sr-90 are really urgent.

$\mathrm{Sr}-90$ is a pure beta-emitter with a maximum energy of $0.5 \mathrm{MeV}$. When using the traditional determination method, chemical pretreatments are needed to separate and purify Sr-90 from other elements by selective precipitations, liquid-liquid extraction (LLE), extraction chromatography (EC) and ion exchange (IE) [8]. One of the widely used precipitation methods is based on the solubility difference of strontium and other cations in fuming nitric acid, which is more accurate than other methods. However, numerous steps are required before separating because the fuming nitric acid is harmful to the

\footnotetext{
^e-mail: xgxu@ustc.edu.cn
}

operator and environment. Other methods such as LLE, EC and IE usually have to wait for about 14 days till the Sr-90 sample reaches equilibrium with the Y-90 [9], and then isolate Y-90 for the indirect determination of Sr-90. The long waiting time of these methods makes them inapplicable to emergency response. In addition, for the IE, the ion exchange resin does not have a higher selectivity for Sr-90 either, so that the larger the sample, the more complex the isolation, because large amount of other elements may interfere separating small amount of strontium. LLE is suitable for the matrix of simple composition. A lot of work has been done to find simpler, faster and more accurate methods. Nowadays, extraction chromatographic resin has been developed for isolation of radionuclides from various kinds of matrix [10-13]. In China, the conventional EC method for analyzing strontium-90 in crops is based on the $G B 11222.1$ - 89 [14] which uses ion-exchange resin with di (2-ethylhexyl) phosphoric acid (HEDHP) to isolate Y-90, counting Y-90 with lowbackground proportional counter, and then the radioactivity of Sr-90 can be evaluated. Horwitz et. al reported that strontium could be extracted in nitric acid using a crown ether [15], dicyclohexano-18-crown-6 (DCH18C6). Right now, this substance is commercially available called Sr•Spec. The resin enables a rapid and simple separation for strontium. It's used more and more widely to separate Sr-90 from different matric, such as milk, soil, water and biological sample [16-19]. However, before passing through the resin a suitable chemical pretreatment is needed to eliminate some interfering nuclides since the resin has saturated adsorption capacity. For milk samples, a large amount of $\mathrm{Ca}^{2+}$ interfere the resin from absorbing 
Sr because of their chemical similarities. Thus special procedures for eliminating $\mathrm{Ca}^{2+}$ should be taken before the sample is passed through the resin. For sediment samples, $\mathrm{Ba}^{2+}$ may be the interference. For biological samples, the organic matter need to be removed before analyzing. In this paper, a rapid method and a modified conventional method for analyzing the Sr-90 with background level in crops are described. The modified conventional method is based on the GB11222.1 - 89 in China. The conventional method for analyzing Sr-90 could not completely remove $\mathrm{Bi}-210$ which interferes the measurement results. Some crops samples have a large amount of lead, bismuth and potassium. In those cases, the conventional method would not work. The rapid method uses liquid scintillation counter (LSC) together with Eichrom Srøspec extraction resin as an agent to separate strontium and applies spectrum analysis [20-22] for measuring the Sr-90 activity. Compared to the similar methods in former literatures, a special co-precipitation method is applied before samples passed through the resin in order to prevent the competition of other ions in the rapid method. The modified conventional method and the rapid method for analyzing Sr-90 in crops cover all the situations of emergency response, daily monitoring.

\section{Experiments}

\subsection{Reagents}

Sr•Spec Resin (particle size: $100-150 \mu m$ ) was obtained from EiChrom Industries, Inc. Nitric and hydrochloric acids of various concentrations were used to dissolute precipitation and other functions. All chemical reagents are of analytical grade quality. The $\mathrm{Sr}-90$ (Sr-90/Y-90 in equilibrium) and $\mathrm{Pb}-210(\mathrm{~Pb}-210 / \mathrm{Bi}-210 / \mathrm{Po}-210$ in equilibrium) as certified standard aqueous solution are obtained from textitEckert \& Ziegler.

\subsection{Instrumentations}

- Quantulus $1220^{T M}$ liquid scintillation counter (PerkinElmer, Inc. USA).

- Low-background proportional counter.

- Centrifugal machine.

- Water bath kettle.

- Analytical balance, $0.1 \mathrm{mg}$ accuracy.

\subsection{Sample pretreatment}

The purpose of sample pretreatment is to obtain a homogenous sample. The crops were collected in China. All the samples were homogenized and dried on a hot plate until constant weight. Afterwards, the samples were ashed to 600 in muffle furnace.

\subsection{Rapid method for Sr-90 separation from crops samples}

Based on the previous researches, it's possible to directly isolate strontium from the crops. This method was investigated and developed here. The mainly procedures of rapid method for analyzing Sr-90 include three parts: radiochemical procedure, source preparation \& measurement, and activity determination. The flow diagram of this method is shown in figure 1 .

\subsubsection{Detailed radiochemical procedures}

After pretreatment, white ashes were obtained. 5 grams crops ashes were taken for the analysis. $30 \mathrm{mg} S r^{2+}$ carrier and $10 \mathrm{mg} Y^{3+}$ were added for chemical yield determination. The ashes were mixed with hydrochloric acid $5 \mathrm{M}$ for leaching about one hour. The insoluble substances were filtered to get clear filtrate. After leaching and filtering, oxalic acid was added to the filtration and $\mathrm{Sr}$ was separated from the other alkaline earth metals by oxalate precipitation at $\mathrm{pH}=4$. Compared to the strontium, a large amount of potassium was in the crops, so oxalic acid was added to the solution to separate strontium from potassium avoiding ion competition. Ion competition may lead to lower chemical yield, which interferes the measuried results. Afterwards, the precipitation was dissolved with $20 \mathrm{~mL} 8 \mathrm{M}$ nitric acid and the solution was passed through the column filled with Eichrom $\mathrm{Sr} \bullet$ Spec resin.

The resin should be soaked in the water for a long time (several hours) to remove impurities before being transferred into the column. The Sr•Spec column was equilibrated with $15 \mathrm{~mL} 3 \mathrm{M}$ nitric acid, and then the sample solution was passed through. The column was washed successively with $20 \mathrm{~mL} 8 \mathrm{M}$ nitric acid and $15 \mathrm{~mL} 3 \mathrm{M}$ nitric acid, $\mathrm{Bi}, \mathrm{Y}, \mathrm{K}, \mathrm{Ca}$ and other elements passed the column, strontium was retained by it. Then $40 \mathrm{~mL}$ deionized water was used to elute Sr-90. It is important to realize the washing solution volume should be controlled, otherwise it would elute strontium as well.

\subsubsection{Source preparation and measurement}

The Sr-90 eluting solution was adjusted to $\mathrm{pH}=9.0$ with ammonium hydroxide. After adding $5 \mathrm{~mL}$ saturated ammonium carbonate, filtering and heating, the yields of strontium were determined by gravimetric method. The precipitation was dissolved with $8 \mathrm{~mL} 0.1 \mathrm{M}$ nitric acid, and then mixed with $12 \mathrm{~mL}$ liquid scintillation cocktail HiSafe TMIII for the measurement using LSC.

The measurement was performed with a Quantulus $1220^{T M}$ counter. This apparatus uses low coincidence bias to reduce the background. The multichannel analyzer (MCA) configuration was set in the Alpha/ Beta option and the pulse shape analysis (PSA) was set to 100 . For the determination of activity, the efficiency calibration for different radionuclides should be performed. A certain amount of Sr-90/Y-90 standard solution with increasing amounts of $\mathrm{CCl}_{4}$ as quenching agent was used. Then the quenching correction curve was 


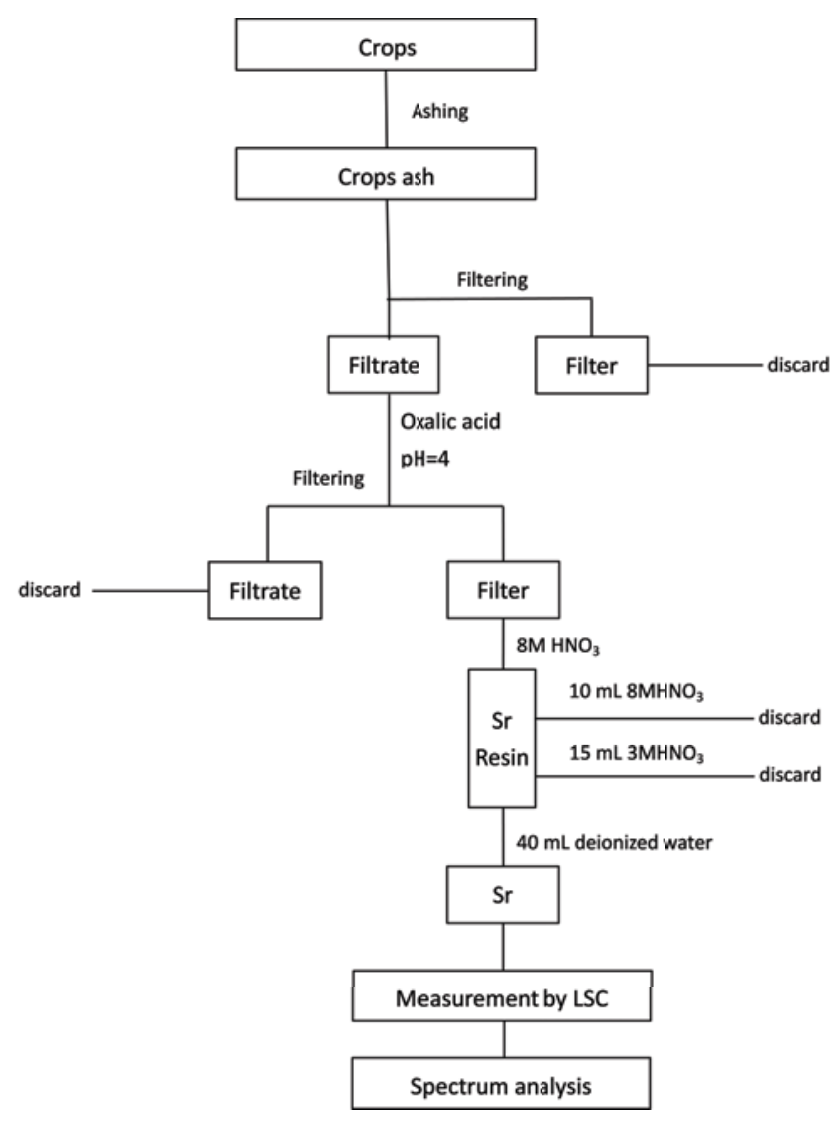

Figure 1: Flow diagram for rapid method determination of Sr-90 in crops.

obtained by using an Eu-152 source. Sr-90 as a $\beta$ radionuclide with high energy normally has little influence on chemical quenching, which keeps the quench correction curve away from obvious changes. Finally, background correction and time correction were performed.

\subsubsection{Activity determination}

LSC is a detector with spectrum properties. Therefore, the spectrum analysis method can be applied to determine the Sr-90 activity. Impurities can be detected when using spectrum analysis method performing different spectra, especially for radionuclides mixed with their isotopes which cannot be separated with chemical treatments. The activities of all the radionuclides can be calculated using spectrum analysis, as long as the standard spectra of all the radionuclides are known.

There are totally 1024 channels in LSC and the Sr-90 spectrum covers from channel range [5th $-600 t h]$. The spectrum obtained from the LSC shows that high energy windows (channel range [150th $-600 t h]$ ) should be focused on to avoid the interference from low energy radionuclides, such as $\mathrm{Pb}-210$. Then spectrum unfolding based on the least squares algorithm (as shown in Eq. 1) is applied to determine the activities. Once all the radionuclides in samples are known, the activity of each nuclide can be easily calculated using the spectrum analysis method, without any chemical treatments to the samples.

$$
Y_{i}=\sum_{n} C_{n} \cdot a_{n, i}
$$

Where $y_{i}$ is the counting number of each channel. $C_{n}$ is the activity of the unknown nucleus $n$ in the mixed sample, and $a_{n, i}$ is nuclide $n$ 's counting responding in the $i t h$ channel.

In this rapid method, the $\mathrm{Sr}$ resin can separate $S r^{2+}$ from other interference substance directly. In an emergency situation, this rapid method can be used to determine the activity of $\mathrm{Sr}-90$ together with other strontium isotopes and oxalic acid cannot be added to the filtrate due to the small sampling. Even though the rapid method works for all the situations, the main problem of this method is that the $\mathrm{Sr}$ resin is really expensive. Regarding to the daily monitoring, a much cheaper resin separating Y-90 can be used for samples in which Sr-90 already reaches equilibrium with the Y-90. Some of these conventional methods using other resins, like the Chinese national standard method for determining Sr-90 activity, may have shortcomings in the presence of large amounts of lead and bismuth. To meet the needs for daily monitoring, a modified method was described as below.

\subsection{Procedures for modified conventional method}

Ion-exchange resin with HDEHP is used in the modified conventional method, together with low-background proportional counter (LLP) to determine the activity of Y-90. 
And then Sr-90 activity is derived from some calculation formulas.

A certain amount of sampling ash was taken for the analysis. 50mg $S r^{2+}$ carrier and $20 \mathrm{mg} Y^{3+}$ were added for chemical yield determination. The ash samples were dissolved by leaching with hydrochloric acid 5M. Oxalic acid was added and the $\mathrm{pH}$ was adjusted to 3 after leached. Yttrium and other alkaline earth elements were precipitated as oxalates, a process in which the potassium and some other interferences were removed. Oxalate precipitation was destructed by ashing and then was dissolved by nitric acid. The filtrate was passed through the HDEHP column to remove $\mathrm{Sr}, \mathrm{Cs}, \mathrm{Ce}$ and other elements. Y-90 was eluted with $6 \mathrm{M}$ nitric acid. $10 \mathrm{mg} \mathrm{Bi}$ carrier was added to the eluent solution to purify Y-90 from bismuth sulfide precipitation. Thereafter, the purification solution was taken to determine the chemical yield by gravimetric method and the activity of Y-90 was measured by a LSC or LLP. The activity of Sr-90 was then determined. The flow diagram for the separation of Y-90 from crops ash is presented in figure 2 .

\section{Results and discussion}

The spiked samples for conventional method were added with $\mathrm{Sr}-90$ standard solution, $\mathrm{Pb}-210$ standard solution and Sr-90 mixed with $\mathrm{Pb}-210$ standard solution respectively and then measured by LSC. The results of the LSC were shown in figure 3. Comparing the spectra in figure 3, the spectrum spiked with $\mathrm{Pb}-210$ obtained from the conventional method has an obvious peak observed between channel range [200th-400th]. The spectra of Sr-90 mixed with $\mathrm{Pb}-210$ standard solution and $\mathrm{Sr}-90$ standard solution are obviously different. Comparison of the three figures implies that $\mathrm{Bi}-210$ was not removed thoroughly in the conventional method which leads to the interference.

The same samples were analysed by modified conventional method and conventional method respectively, the results were shown in figure 4 and figure 5. The spectrum from the conventional method has obvious peaks near the center of the energy channels, but the spectrum using modified measuring method does not. The results imply that the modified method can separate Bi from the sample to some extent.

Figure 6 and figure 7 show the spectra of the crops sample and the spiked crops sample operated by rapid method. A good consistency with the strontium standard spectrum can be observed. From figure 4 to figure 6, there are obvious peaks in low energy channels when the sample has low-level radioactive contamination. It may be caused by the interference from environmental radon and its daughters. That is why we shifted to the high energy windows while using the spectrum analysis method to calculate the activity of Sr-90.

\subsection{Spectrum method for determining Sr-90 activity}

Figure 8 shows a spectrum corresponding to Sr-90 and Y90 in secular equilibrium. Both Sr-90 and Y-90 present a continuous spectrum from 0 to Emax since they are pure $\beta$ nuclides. Y-90 has a shorter half-life so that Sr-90 decays to Y-90 rapidly leading to an overlap between their individual spectra even though Y-90 was eliminated completely. Spectrum analysis method can be used to eliminate the interference from Y-90. Appropriate windows should be chosen based on the rules that window A contains pulses both from Sr-90 and Y-90 whereas window B only contains Y-90 pulses.

From Y-90`s standard spectrum in figure 3, which spiked Sr-90 standard solution obtained from conventional method, window B could be chosen from channel range [600th-850th], in which only Y-90 pulses exist. With respect to window $\mathrm{A}$, the window was selected with the purpose of getting a high efficiency for Sr-90 and low interference from other nuclides. Because of the influence from environmental radon and cosmic rays, the window A could be chosen from channel range [150th-600th], which gets rid of low channel parts. The expression for calculating the Sr-90 activity is shown as below:

$$
\begin{aligned}
C_{A} & =A_{1} \epsilon+A_{2} \phi_{A} \\
C_{B} & =A_{2} \phi_{B} \\
A_{1} & =\frac{C_{A}-\frac{\phi_{A}}{\phi_{B}} C_{B}}{\epsilon}
\end{aligned}
$$

Where $A_{1}$ denotes the Sr-90 activity in the sample, $\epsilon$ is the counting efficiency of $\mathrm{Sr}-90$ in window $\mathrm{A}, A_{2}$ is the activity of Y-90 in the sample, $C_{A}$ and $C_{B}$ are the count rate in the window $\mathrm{A}$ and window $\mathrm{B}$, and $\phi_{A}$ and $\phi_{B}$ are the counting efficiencies of $\mathrm{Y}-90$ in window $\mathrm{A}$ and window $\mathrm{B}$. The value of these parameters could be obtained in such a way described as follow. Sr-90 was separated from a certain standard solution and then this sample was measured several times after the separation date. The count rate in the window $\mathrm{A}$ and window $\mathrm{B}$ at different time would be recorded. And then least square method was used to determine each parameter. We chose the optimal window A to be in the channel range [150th-600th] and window B to be in the channel range [600th $-850 t h]$, and the value of the Sr-90 counting efficiency $\epsilon$ was $88.4 \%$ in window A and the ratio of efficiencies for $\mathrm{Y}-90$ in the two windows $\frac{\phi_{A}}{\phi_{B}}$ was 0.458 .

\subsection{Minimum detectable activity (MDA)}

MDA is one of the most important value to estimate the radioactivity method. The formula of MDA was evaluated as follows:

$$
\operatorname{MDA}(B q / \mathrm{kg})=\frac{2.71+4.65 \sqrt{B}}{E T Y m f}
$$

Where B is the total number of background measured separately with the same counting time, E is the counting efficiency, $\mathrm{T}$ is the sample counting time, $\mathrm{Y}$ is the chemical yield, $\mathrm{m}$ is the mass of sample and $\mathrm{f}$ is the correction coefficient. According to the equation, the value of MDA for rapid method is $2.32 \mathrm{~Bq} / \mathrm{kg}$. When the calculation method 


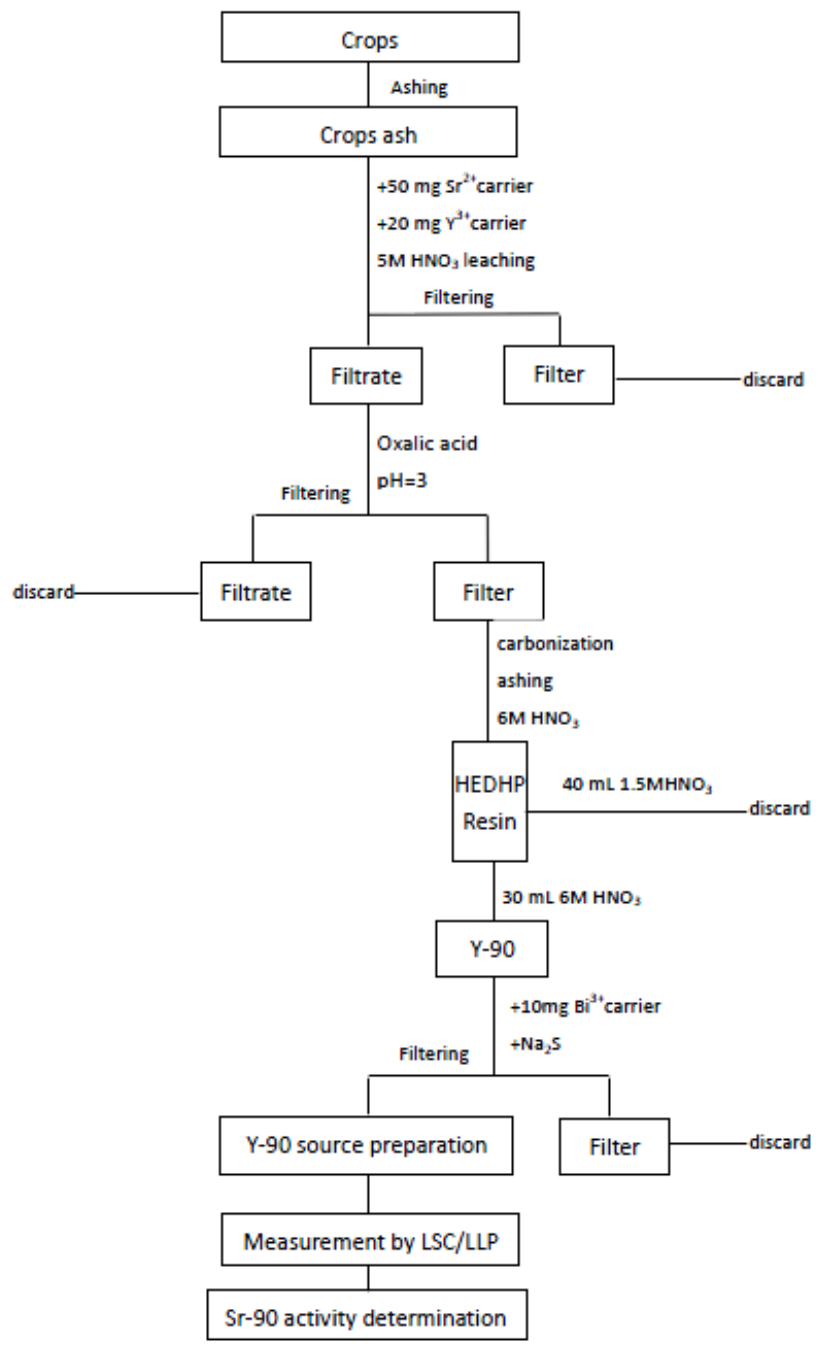

Figure 2: Flow diagram for modified conventional method of Y-90 in crops

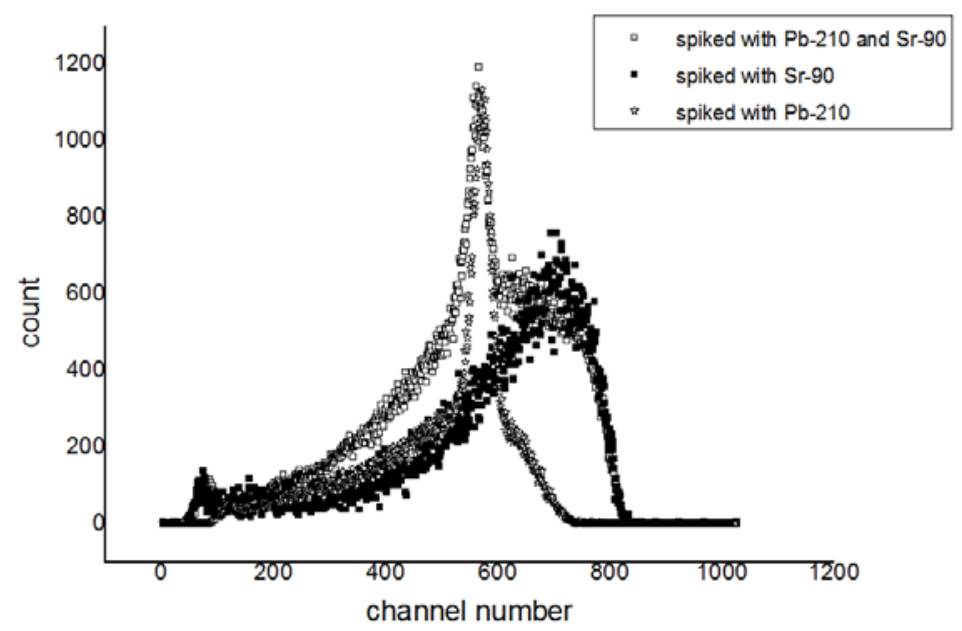

Figure 3: Spectra of spiked samples obtained from the conventional method. 


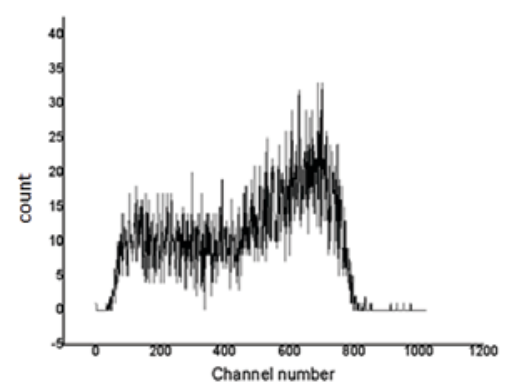

Figure 4: Spectrum of crops ashes obtained from the modified conventional method.

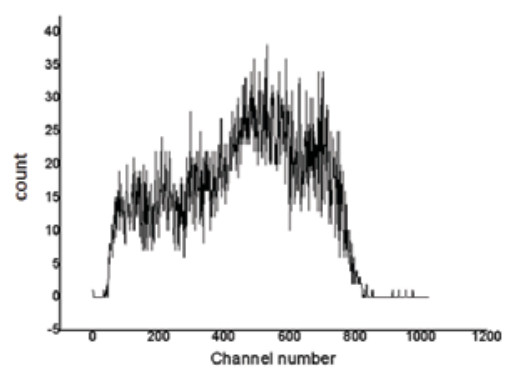

Figure 5: Spectra of spiked samples obtained from the conventional method.

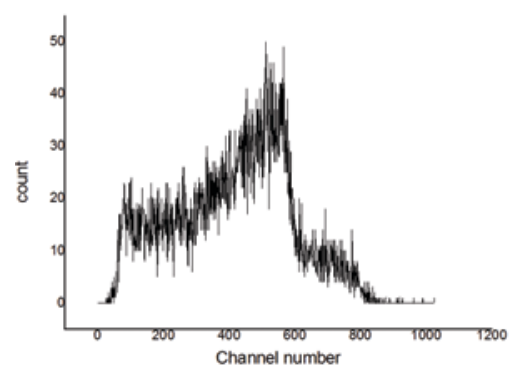

Figure 6: Spectrum of crops ashes obtained from the modified conventional method.

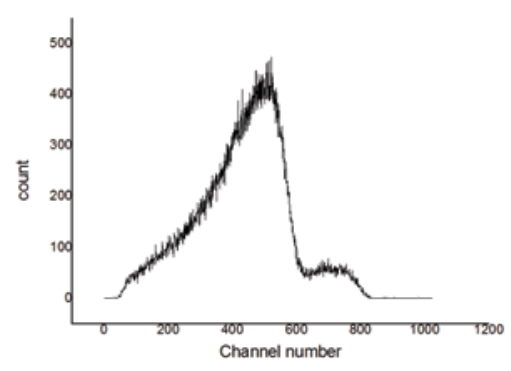

Figure 7: Spectra of spiked samples obtained from the conventional method.

is applied to the environmental samples, the MDA should be estimated for each sample due to the different factor.

As shown in Table 1, the results of the rapid method are slightly and systematically smaller than those of the modified conventional method. The reasons for this may be the modified conventional method for the sample with a large amount of bismuth not eliminating bismuth completely in the error-allowed range and the system error between different detectors. However, the difference of the
Table 1: Comparison of analytical results of Sr-90 activity concentration. (CM: conventional method; MCM: modified conventional method; RM: rapid method)

\begin{tabular}{llll}
\hline Sample & $\mathrm{CM}(\mathrm{Bq} / \mathrm{kg})$ & $\mathrm{MCM}(\mathrm{Bq} / \mathrm{kg})$ & $\mathrm{RM}(\mathrm{Bq} / \mathrm{kg})$ \\
\hline $\mathrm{A}$ & $23.0 \pm 2.5$ & $18.0 \pm 1.8$ & $16.0 \pm 1.7$ \\
$\mathrm{~B}$ & $8.0 \pm 0.9$ & $6.8 \pm 0.7$ & $5.4 \pm 0.5$ \\
$\mathrm{C}$ & $11.0 \pm 1.2$ & $9.1 \pm 1.0$ & $7.2 \pm 0.7$ \\
$\mathrm{D}$ & $12.0 \pm 1.3$ & $8.9 \pm 0.9$ & $7.4 \pm 0.8$ \\
E & $8.3 \pm 1.1$ & $4.7 \pm 0.8$ & $4.1 \pm 0.4$ \\
F & $7.9 \pm 1.2$ & $3.4 \pm 0.6$ & $3.4 \pm 0.3$ \\
G & $14.2 \pm 1.7$ & $11.1 \pm 1.0$ & $10.5 \pm 1.1$ \\
H & $4.0 \pm 0.8$ & $4.0 \pm 0.9$ & $3.6 \pm 0.3$ \\
\hline
\end{tabular}

values between the modified method and rapid method are actually considered to be acceptable.

\section{Conclusions}

The rapid method proposed in this paper for chemical separation and purification of Sr-90 was found to be simpler, faster and more accurate than the conventional method. The activity of Sr-90 can be calculated without considering Y-90, so it no longer requires waiting for Sr-90 and Y-90 reaching equilibrium. As a result, the whole analysis procedure can be accomplished within one day. Furthermore, Sr-89 and Sr-90 can be detected simultaneously using this spectrum analysis method,so it can be used in nuclear emergency monitoring. For daily monitoring with large amount of samples and low radioactivity, the much cheaper modified conventional method would be a better choice. The two methods do cover different situations.

\section{Acknowledgements}

The work was funded in part by a grant from National Natural Science Foundation of China (No.11575178).

\section{References}

[1] H. Isnard, M, Aubert, P, Blanchet, et al. Spectrosc Spect Anal. 61(2), 150-156 (2006)

[2] C. Landstetter, G. Wallner. J Environ Radioactiv. 87(3), 315-324(2006)

[3] I. Lopes, M. J. Madruga, A. Mourato, et al. J. Radioanal. Nucl. Chem. 87(3), 315-324 (2006)

[4] E. Kabai, L Hornung, B. T. Savkin, et al, Sci. Total Environ. 410, 235-240 (2011)

[5] P. Neis, R. Hille, M, Paschke, et al. Forensic Sci Int. 99(1), 47-51(1999)

[6] E. P. Hardy, M. W. Meyer, J. S. Allen, L. T. Alexander, Nature 219. 584-587 (1968)

[7] F. Goutelard, R. Nazard, C. Bocquet, et al. Radiat. Isotopes. 53(1), 145-151 (2000)

[8] N. Vajda, C. K. Kim. Radiat. Isotopes. 68(12), 23062326 (2010)

[9] M. Heilgeist. Radioanal Nucl Chem. 245(2), 249-254 (2000) 


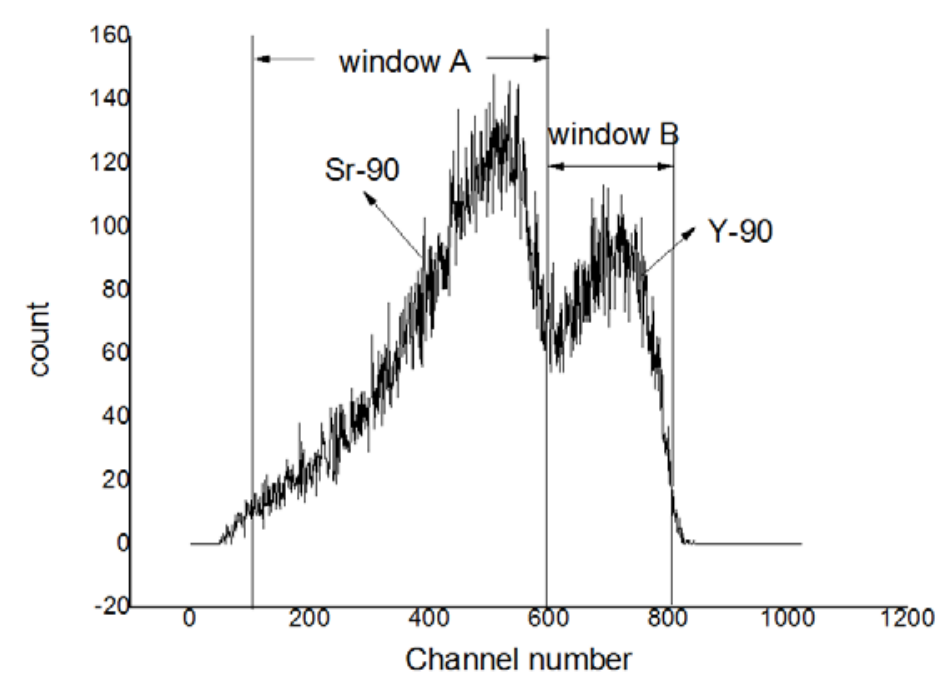

Figure 8: Spectrum of Sr-90/Y-90 secular equilibrium standard solution

[10] Z. Grahek, N. Zecevic, et al, Anal Chim Acta. 399, 237 (1999)

[11] IAEA Tehnical report series 295. 70-89 (1989)

[12] Z. Grahek, I. Eskinja, K. Kosutic, et al. Anal Chim Acta. 379, 107 (1999)

[13] Z. Grahek, K. Kosutic, S. Lulic, et al. J Radioanal Nucl Chem. 242, 33 (1999)

[14] Ministry of Environmental Protection of China, National Standard GB 11222.1-89 (1989) (in Chinese)

[15] R. Bojanowski, D. Knapinska-Skiba. J Radioanal Nucl Chem. 138(2), 207-218 (1990)

[16] R. Bojanowski, Z. Radecki, M. J. Campbell, K. I. Burns, A. Trinkl. IAEA-326 and 327, IAEA Vienna (2001)
[17] S. Brun, S. Bessac, D. Uridat. B, Boursier. J Radioanal Nucl Chem. 252(2), 191-197 (2002)

[18] ASTM Standard Test Method for Strontium-90 in water. D5811-08 (2008)

[19] E. Blasius, W. Klein. J Radioanal Nucl Chem. 89(2), 307-310 (1985)

[20] L. J. Pan, Z. Chen, G. B. Yu, et al. Nuclear Electronics \& Detection Technology. 34(7), 812-815 (2014) (in Chinese)

[21] M. Heilgeist. J Radioanal Nucl Chem. 245(2). 249254 (2000)

[22] K.H. Hong, Y. H. Cho, M. H. Lee, et al. Radiat. Isotopes. 54. 299-305 (2001) 